\title{
Injury data in British Columbia: policy maker perspectives on knowledge transfer
}

\author{
C Mitton, PhD (1, 2); YC MacNab, PhD (2,3); N Smith, MA (1); L Foster, PhD (4)
}

\begin{abstract}
Provincial and regional decision makers in the injury prevention field were interviewed in British Columbia (B.C.) to obtain their views about best processes for the transfer or dissemination of relevant data. These decision makers $(n=13)$ indicated that data should provide them with a holistic and comprehensive picture to support their decision processes. In addition, they felt information about injury types and rates should be linked backward to determinants or causes and forward to consequences or outcomes. This complete chain of data is needed for planning and evaluating health promotion interventions. It was also felt that data providers needed to devote more effort to fostering effective receptor capacity, so that injury prevention professionals will be better able to understand, interpret and apply the data. These findings can likely be generalized to other jurisdictions and policy areas, and offer additional insight into the practicalities of knowledge transfer and exchange in researcher/decision maker partnerships.
\end{abstract}

Keywords: burden of injury, knowledge transfer, knowledge utilization, policy, injury prevention

\section{Introduction}

Much effort can and has been invested in generating data about the impact and burden of chronic diseases in Canada, but do we know how best to utilize these data in policy and practice? Knowledge transfer ${ }^{\mathrm{i}}$ (KT) refers to a process whereby information is made available to decision makers through interactive engagement. Over the past decade, researchers and policy makers have described what inhibits or encourages $\mathrm{KT}^{1-5}$ Based on these findings, guidance about mechanisms or strategies for effective implementation of KT has been published. ${ }^{6-7}$ Common recommendations include establishing ongoing collaborative relationships between the researcher and decision maker,;,8-9 fostering appropriate attitudes, values, culture and capacity within health care organizations; ${ }^{3}$ and offering clear and timely communication in a shared language appropriate to the target audience. ${ }^{2,6,10}$ However, it remains important to pursue a more substantial evidence base around KT practices to ensure research and data-collection efforts are directed appropriately. Empirical case studies with actual datasets in particular contexts, such as the one reported in this paper, should advance our understanding and may offer potential for immediate improvements to practice. While the findings here relate most directly to those interested in injury prevention and health promotion, the issues generated from this study should also be applicable to KT for chronic diseases as well as in health care contexts.

\section{Methods}

Data collection involved semi-structured interviews with 13 key decision makers with direct knowledge of injury prevention policy in B.C. The interview schedule is appended. The following organizations were represented in the sample: the B.C. Ministry of Health (recently divided into the Ministry of Health Services and a separate new Ministry of Healthy Living and Sport), the Provincial Health Services Authority (PHSA) and two of the five regional health authorities (RHAs). Interviewees were primarily senior to middle managers, with job titles such as Executive Director, Director, Manager or Project Lead. These individuals would typically be responsible for broad planning, priority setting and/or evaluation functions. The interviews were conducted in June and July 2005.

The interviewer provided each informant with sample data to look over (see Tables 1 and 2, and Figure 1, as examples). Informants were then asked general questions about what sources of data they currently accessed and to provide feedback about the sample data sets. Each interview was audio recorded (with permission) and transcribed. Analysis proceeded using the constant comparison method, i.e. themes and sub-themes were developed

i For details see: http://www.researchtopolicy.ca/whatwehavelearned/develop_approach.asp. Related terms include knowledge translation, knowledge exchange, knowledge utilization, and research dissemination.

Author References

1 Faculty of Health and Social Development, University of British Columbia Okanagan

2 Child and Family Research Institute of British Columbia

3 Dept. of Health Care and Epidemiology, University of British Columbia

4 Faculty of Human and Social Development and Department of Geography, University of Victoria

Correspondence: Craig Mitton, PhD, Assistant Professor, Faculty of Health and Social Development, University of British Columbia Okanagan, 3333 University Way, Kelowna, BC V1V 1V7, Tel: 250-807-8704, Fax: 250-807-8085, Email: craig.mitton@ubc.ca 
inductively. ${ }^{11}$ Written notes prepared by the interviewer provided an additional source of data. Ethics approval was granted by the University of British Columbia Behavioural Research Ethics Board.

\section{The policy environment for injury prevention in B.C.}

The five RHAs have been given the responsibility to develop and implement plans and programs for injury prevention. They must determine how much of a priority to give to injury prevention compared to other possible initiatives, assess the suitability and effectiveness of program options, engage other sectors where appropriate and evaluate the success and relative costs and benefits of their efforts. The RHAs are at varying stages of development in injury prevention policy. The PHSA provides support with data gathering, analysis and knowledge translation activities and is an important source of data on patient safety, particularly with respect to adverse drug events, nosocomial infections and radiation-related injury.

The role of the B.C. provincial government is primarily one of stewardship. The province also leads surveillance and monitoring efforts. The Health Authority Division of the Ministry of Health Services negotiates performance agreements with the RHAs. These presently include benchmarks for falls and, generally speaking, are meant to hold the RHAs to public account for their outcomes, based on the resources allocated to them. The Healthy Children, Women and Seniors Branch (recently transferred to help create the new Ministry of Healthy Living and Sport) is the primary policy making and advisory centre for the provincial government on injury matters, along with recommendations from the Provincial Health Officer.

The Provincial Health Officer, who now has functions in both the Ministry of Health Services and the new Ministry of Healthy Living and Sport, has provided assistance to RHAs in setting their benchmarks related to falls and has produced a special report on falls among the elderly that: 1) outlines the magnitude of this issue; 2) measures the impact on the health care system; and
3) provides recommendations for evidencebased prevention strategies. ${ }^{12}$ Each RHA must apply these in its own context, of course. Further research and data-collection efforts, in support of developing policy recommendations, have been undertaken since 1997 by the British Columbia Injury Research and Prevention Unit (BCIRPU), located at the Children's and Women's Health Centre of British Columbia. These roles were outsourced following the dismantling of the Ministry's own internal Office of Injury Prevention in 2001. The BCIRPU is expanding its data sources by developing agreements with the Workers' Compensation Board (i.e. WorkSafeBC), Statistics Canada, the Canadian Institute for Health Information (CIHI), the Insurance Corporation of British Columbia (ICBC), the RCMP and BC Ambulance Services.

Other key stakeholders (e.g. Red Cross, RCMP) are involved through such mechanisms as the BC Injury Prevention Leadership Network, a provincial steering committee meant to provide guidance and advice on broad policy directions. Still other entities, such as the BC Aboriginal Health Network, the BC Sport \& Recreation Injury Free Advisory Committee and the BC Falls Prevention Coalition, also exist. The BC Healthy Living Alliance, a consortium of primarily chronic disease organizations also plays a supporting role in issues related to healthy living, including injury prevention. The province, PHSA and RHAs are aware of the interest of these groups, but are not always fully aware of their role or how they might link to other stakeholders. In short, the policy environment pertaining to injury prevention in B.C. is somewhat fragmented, consisting of multiple departments, agencies and organizations.

\section{Injury data used in the research}

Within this policy environment, researchers at the University of British Columbia have been working on a set of statistical methods to further delineate injury data in this province. This case study is part of an ongoing research program, entitled Burden of Injury in BC and Its Local Communities: Information and Evidence for Communitybased Prevention Strategy, Health Policy and Service Provision (short-titled Burden of Injury in BC), currently funded by the Canadian Institutes of Health Research. The main objectives of the Burden of Injury in $B C$ project are: 1) to develop a synthesis of analytic methods for a systematic burden-of-injury research framework that encompasses space-time surveillance monitoring, burden assessment, risk assessment, research dissemination and knowledge translation; and 2) to apply these methods to examine burden-of-injury mortality and disability in B.C. and its local communities. ${ }^{13-14}$

During the policy maker interviews, informants were presented with sample tables of burden-of-injury profiles for each of the five RHAs and for B.C. as a whole. This included mortality and hospitalization counts and rates for major causes of injuries, stratified by gender and based on 1991 to 2000 population-based administrative data. ${ }^{13-14}$ Tables 1 and 2 present the corresponding cause-specific statistics on burden-of-injury, measured by years of life lost to premature death (YLLs), years of life lived with disability (YLDs), and disability adjusted life years (DALYs). ${ }^{15}$ These burden-of-injury measures were derived based on observed injury mortality and hospitalization counts for the five health authorities and for B.C. as a whole for the calendar years of 1991-2000. ${ }^{14}$ The YLL, YLD and DALY estimates in Tables 1 and 2 were derived using disability weight and duration estimates from the 1990 Global Burden of Disease study, specified with zero age weighting and an annual $3 \%$ discount rate. ${ }^{14-15}$ Note that the DALYs are "health gap" measures that allow the combined impact of mortality and morbidity to be incorporated and assessed simultaneously. The DALY measures were developed under the Global Burden of Disease project, ${ }^{15}$ as population health indicators for public health assessment and as a "currency" for cost-effectiveness analysis with respect to priority setting and evaluation of health interventions. ${ }^{14-15}$

Informants were also asked to view two sample maps that depicted annual iatrogenic injury risk estimates for children and youth aged 1 to 19 years for 16 geographic subdivisions of the health authorities, i.e. health service delivery 
areas (HSDAs). One of the maps, presented in Figure 1, highlights the HSDAs with high/low iatrogenic injury risks for the male population. Bayesian estimates of annual relative risks, quantified by the ratios of HSDA rates over a B.C. average were derived in order to prepare this map. ${ }^{13,16}$ An HSDA was identified as having a high (or low) iatrogenic injury risk if the $95 \%$ interval estimates (i.e. the upper and lower limits) of the relative risk were above (or below) one. ${ }^{13,16}$

\section{Results}

Findings from the interviews relate to both the content and the processes that should lead to effective dissemination and uptake of injury data by researchers and decision makers in B.C. The content of data sets needs to provide a rounded perspective that addresses both injury causes and consequences. Dissemination processes should be targeted to specific audiences and recognize that individuals and organizations may have differing degrees of capacity to use information.

\section{Data content}

The interviewees were familiar with and have used DALY measures for health planning purposes, though one suggests that “[we] probably don't use them as much as we should, but we definitely use them" [\#3]. However, they suspect that other audiences are less familiar with this measure and would be better versed with indicators like morbidity and hospitalization rates. One interviewee suggested that DALYs might not have as much traction as other measures, because they are not linked or aligned to the "strategic goals of the health system” [\#1]. Health system managers in B.C. are not held accountable by their performance agreements with the province for changes in this measure as they are for some other targets. This suggests, as do findings reported below, that institutional or systemic barriers are important influences on the KT process.

These decision makers wanted rich data so that they could view policy issues in their full complexity. They wanted a data "chain" so that they could look both forward and backward from specific injury events. Currently, much factual and descriptive data are available about injury incidence and prevalence, i.e. what types of injury occur and the demographics of those injured. This is useful, but these policy makers also wanted to know about determinants and contexts of injury, i.e. they sought information to help them understand why circumstances in their own communities are the way they are, and whether there are any unique local concerns, needs or circumstances that ought to be acknowledged and addressed.

Decision makers also wanted to be able to look at data from many possible angles and to consider injury findings in light of a range of variables. Demographics of the people involved, times and locations of incidents, and conditions in the social or physical environment are among the factors that might be relevant in understanding and explaining the local injury picture. This kind of information would likely come from a retrospective investigation and a description of each incident.

I think what I would like to be able to see is, okay, what are the various types of falls? What are the causes and the impacts and who is most at risk? And so, we need to know first of all, who's falling, when are they likely to fall and what are they doing when they are falling? [\#1]

We know motor vehicle crashes [are] number one, but we need to break it down. What does that actually mean? Is it commercial drivers, is it alcohol, is it due to lack of seatbelt use? [\#3]

In addition, determinant and context data are necessary in order for planners to decide how best to intervene to solve the problem and carry out evaluative efforts. The informants had a particular interest in these functions and desired data that would assist them in effective planning and evaluation efforts.

I think it's a matter of articulating, understanding the issues and the determinants, and how the solutions come in to make a difference, because the solution is obviously predicated on an understanding of the determinants. [\#1]
When I think about data, I'm not thinking about rate of; I'm constantly linking it back to... to evidence for interventions and the data related to that, and linking it back then to my population and the data of my population so that I can develop an appropriate intervention. It's so important to link health data with evidence for effective interventions. [\#9]

Policy makers also wanted data about the aftermath of injury. This would require following injury cases over time and gathering information about medium- to long-term consequences at the individual and population levels (including full costing). The "business case" for investing in injury prevention depends upon good knowledge of outcomes.

The outcome-figuring out, do they recover; do they go back to work; are they able to walk; are they able to resume previous activities or does it precipitate a downward spiral in their health? And, as so often with the elderly, that's what happens. You know, we can talk about ActNow [a provincial government health promotion initiative], we can talk about healthy living and exercise and so forth [but] often, a setback like that for an elderly person is extremely detrimental to their health. [\#1]

For policy decisions, we've really got to turn it [injury data] into a bit of a business case and a business case model [for investment in prevention interventions]. ... We're trying to convince government that this is important to pay attention to, because this burden of illness on the system is really a surrogate for the costs we're spending in the healthcare system. ... So, my advice would be to take this information and convert it into something that the decision makers on the resource allocation ledger can understand, interpret and begin to believe that, if I invest in this other end of the continuum, I'm going to take some pressure off the far end-a very compelling argument. ... For them to know what percentage of spending 
contributes from the overall injuries on sports injuries, drowning and burns, they can then start targeting their response. [\#2]

However, according to these informants, the data that would flesh out this data chain are unavailable or difficult to obtain in B.C.

\section{Processes for data dissemination}

Several comments directly address dissemination processes. A range of ideas was raised by the interviewees (e.g. using "knowledge brokers") about how the information embedded in indicator data might best be transferred to these decision makers, but all emphasized that the credibility of who transfers the knowledge was important and that the knowledge be tailored to each specific target audience. Those who set priorities, for instance, may need different data about the costs and outcomes of injury than those who are responsible for detailed program evaluation.

It's really dependent on what you're using it for and who your audience is. From a decision making point of view, if you understand what they say, then it doesn't matter. You can look at raw numbers or raw tables and you can gather the information that you need to make the decision. But it's more in putting a rationale, or putting a business case forward or that kind of stuff where you need to have the whole array of tools. [\#5]

The visual depiction of data in map format was seen as having appeal for non-experts (e.g. board or senior-management decision makers). "If you want to disseminate this information widely, to people in policy or practitioners, or whoever you want to digest this information, I think the maps are more effective." [\#1] Maps were seen as a quick way to transmit information to audiences that are pressed for time and that are unable or unwilling to read lengthy reports or academic journal articles. In this way, they are comparable to the briefing notes or fact sheets touted by organizations like the Canadian Health Services Research Foundation (www.chsrf.ca) as an effective means of reaching senior decision makers with research-based evidence.

Maps were seen as particularly valuable for presenting comparisons. "You can look at it on the page and compare [yourself with] other people. ... It's nice to see where you are pictorially in relation to the rest of the province." [\#7] Interestingly, one respondent from the provincial government framed the issue of comparison in the context of the province's specific intention to encourage competition among the health authorities:

I like the whole idea of spatial mapping around injuries; I think that's the way we should go ... we're sort of setting up the health authorities to be quite competitive, so we need to be able to map which health authorities have got the highest injury rates and relative risks of certain injuries across the province. [\#3]

Maps were identified as a valuable communication tool and a good way to make a point: "If I'm going to be using [data] for the purposes of communication to others, ok, I'll go for the spatial mapping." [\#6] of course, analysis and interpretation are embedded in the argumentative use of data in any form:

TABLE 1

Years of life lost to premature death (YLL), years of life lived with disability (YLD) and disability adjusted life years (DALYs) in British Columbia males, by health authority and cause of injury, 1991-2000 cumulative total

\begin{tabular}{|c|c|c|c|c|c|c|}
\hline \multirow{3}{*}{ Injury } & \multicolumn{6}{|c|}{ Males } \\
\hline & \multicolumn{5}{|c|}{ Health authorities } & \multirow[b]{2}{*}{ B.C. } \\
\hline & Interior & Fraser & $\begin{array}{l}\text { Vancouver } \\
\text { coast }\end{array}$ & $\begin{array}{l}\text { Vancouver } \\
\text { Island }\end{array}$ & $\begin{array}{l}\text { Northern } \\
\text { B.C. }\end{array}$ & \\
\hline \multicolumn{7}{|c|}{ Years of life lived with disability (YLD) } \\
\hline Road traffic injuries & 8500 & 10096 & 5489 & 5951 & 4656 & 34692 \\
\hline Other transport injuries & 1691 & 1424 & 607 & 1039 & 1186 & 5946 \\
\hline Falls & 3848 & 5526 & 3630 & 3564 & 2037 & 18604 \\
\hline Burns/fires/scalds & 1121 & 1370 & 1119 & 982 & 1033 & 5624 \\
\hline Drowning & 174 & 336 & 181 & 141 & 120 & 952 \\
\hline Sports injuries & 562 & 766 & 467 & 470 & 275 & 2540 \\
\hline Other unintentional injuries & 8622 & 9826 & 5921 & 7118 & 6088 & 37576 \\
\hline Suicide and self-inflicted injuries & 1008 & 1925 & 1183 & 1104 & 602 & 5822 \\
\hline Other intentional injuries & 1219 & 2126 & 1687 & 1290 & 1116 & 7438 \\
\hline
\end{tabular}


TABLE 1 (continued)

Years of life lost to premature death (YLL), years of life lived with disability (YLD) and disability adjusted life years (DALYs) in British Columbia males, by health authority and cause of injury, 1991-2000 cumulative total

\begin{tabular}{|c|c|c|c|c|c|c|}
\hline \multirow{3}{*}{ Injury } & \multicolumn{6}{|c|}{ Males } \\
\hline & \multicolumn{5}{|c|}{ Health authorities } & \multirow[b]{2}{*}{ B.C. } \\
\hline & Interior & Fraser & $\begin{array}{c}\text { Vancouver } \\
\text { coast }\end{array}$ & $\begin{array}{l}\text { Vancouver } \\
\text { Island }\end{array}$ & $\begin{array}{l}\text { Northern } \\
\text { B.C. }\end{array}$ & \\
\hline \multicolumn{7}{|c|}{ Years of life lost to premature death (YLL) } \\
\hline Road traffic injuries & 19008 & 19357 & 9565 & 9962 & 11081 & 68973 \\
\hline Falls & 3295 & 4284 & 5125 & 3702 & 1042 & 17448 \\
\hline Burns/fires/scalds & 1112 & 968 & 912 & 720 & 830 & 4542 \\
\hline Drowning & 2024 & 2130 & 1781 & 1905 & 1337 & 9177 \\
\hline Sports injuries & 138 & 78 & 233 & 28 & 64 & 541 \\
\hline Other unintentional injuries & 2009 & 1550 & 1189 & 1595 & 1255 & 7598 \\
\hline Suicide and self-inflicted injuries & 16335 & 21106 & 19110 & 15252 & 7861 & 79664 \\
\hline Other intentional injuries & 2420 & 4764 & 4720 & 2116 & 2021 & 16040 \\
\hline \multicolumn{7}{|c|}{ Disability adjusted life years (DALYs) } \\
\hline Road traffic injuries & 27508 & 29452 & 15054 & 15913 & 15737 & 103664 \\
\hline Other transport injuries & 4473 & 4218 & 2510 & 3389 & 4099 & 18689 \\
\hline Poisoning & 5994 & 18269 & 27127 & 10249 & 3289 & 64928 \\
\hline Falls & 7143 & 9810 & 8755 & 7266 & 3079 & 36053 \\
\hline Burns/fires/scalds & 2233 & 2338 & 2031 & 1701 & 1863 & 10166 \\
\hline Suicide and self-inflicted injuries & 17343 & 23031 & 20293 & 16356 & 8463 & 85486 \\
\hline Other intentional injuries & 3638 & 6890 & 6407 & 3406 & 3137 & 23478 \\
\hline
\end{tabular}


TABLE 2

Years of life lost to premature death (YLL), years of life lived with disability (YLD) and disability adjusted life years (DALYs) in British Columbia females, by health authority and cause of injury, 1991-2000 cumulative total

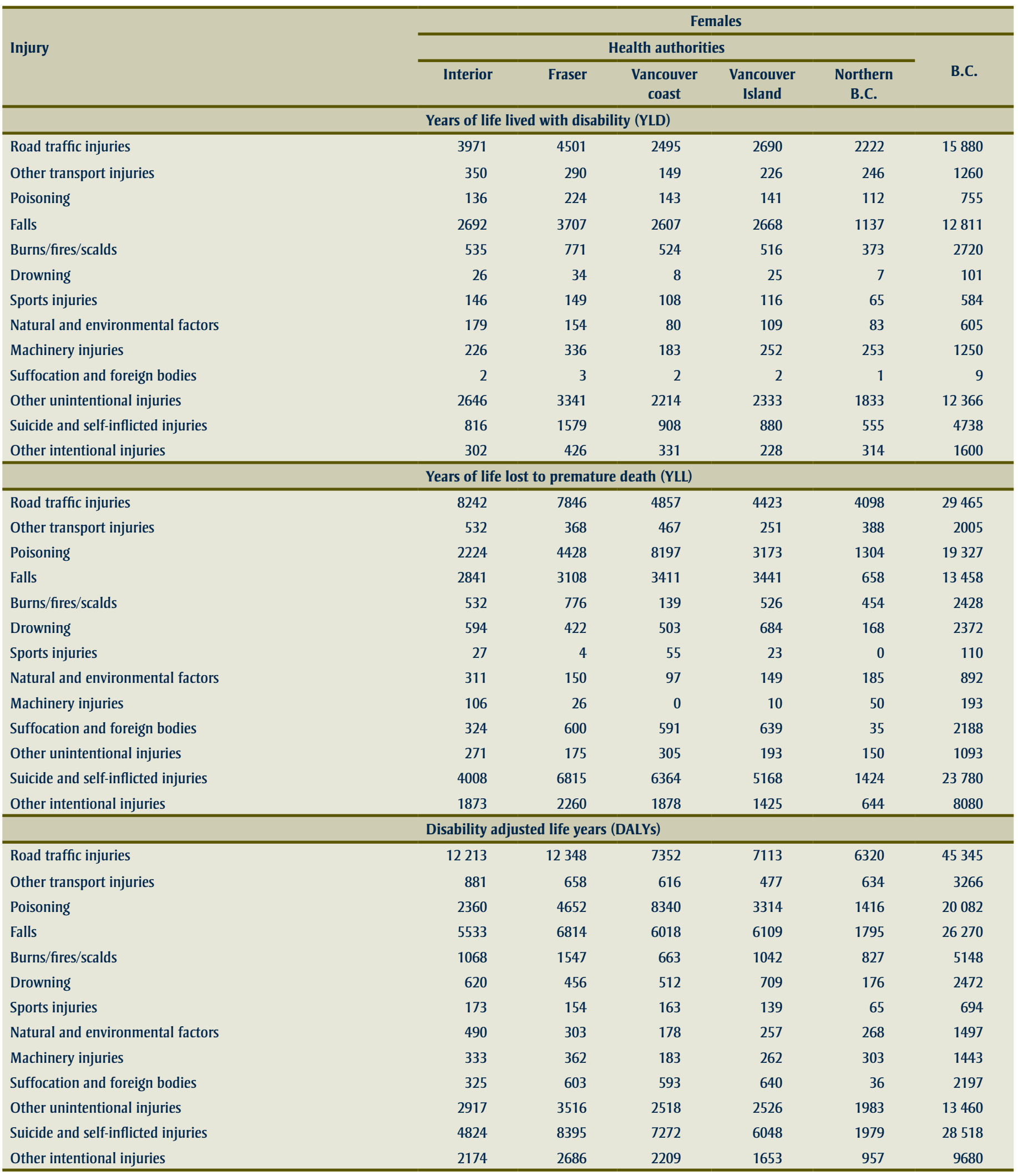


FIGURE 1

High and low iatrogenic injury risk estimates for male British Columbia children and youth aged 1 to 19 years, by health service delivery area, 1991-2000
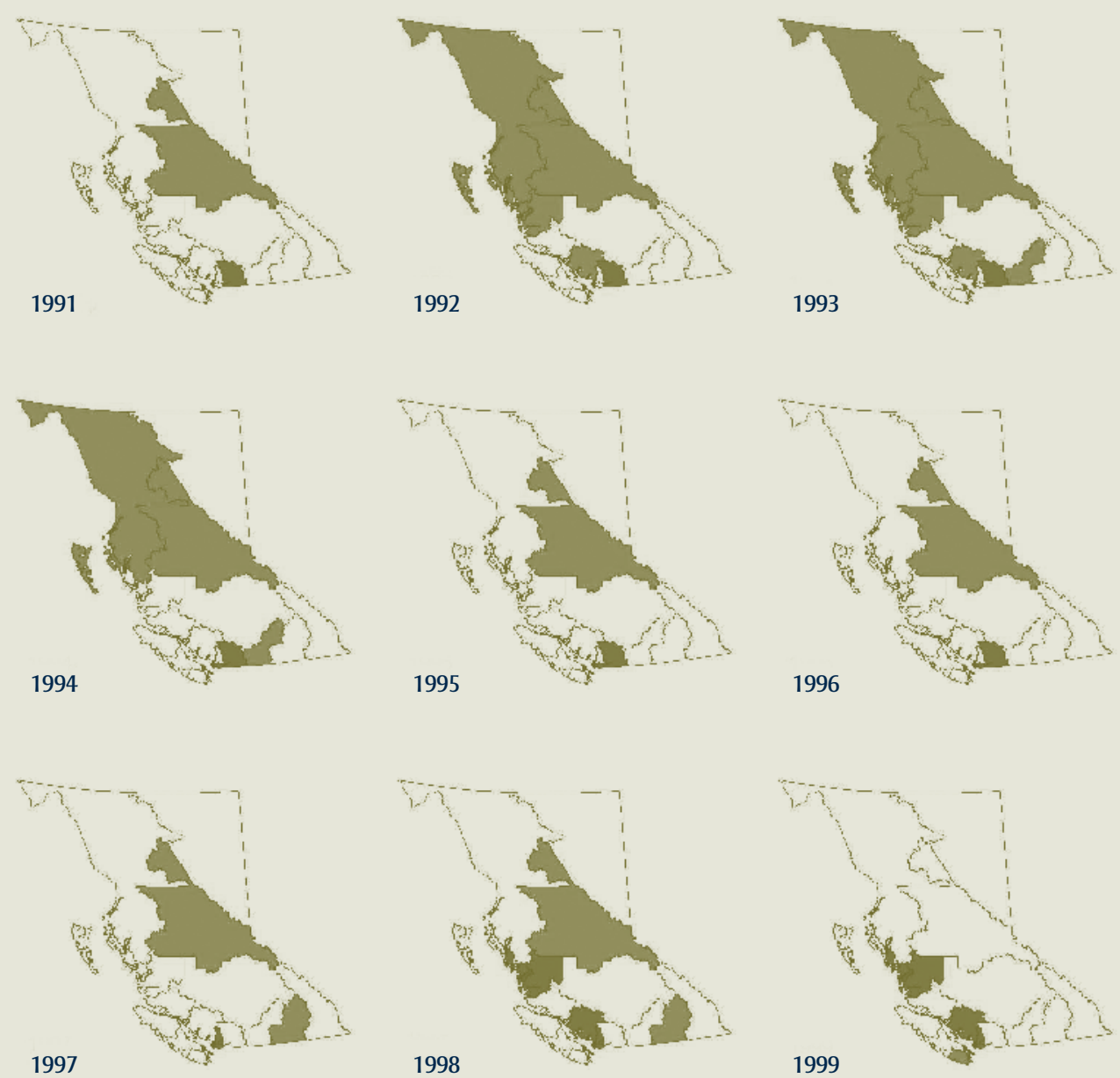

1997

1998

1999

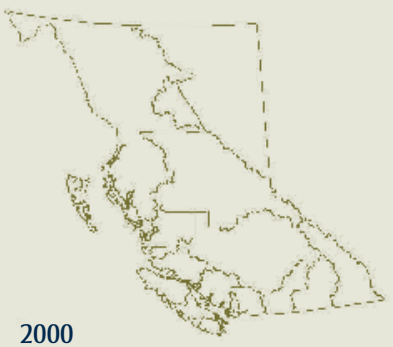

High relative risk

Low relative risk

\section{Relative Risk}


FIGURE 2

The injuries "data chain"

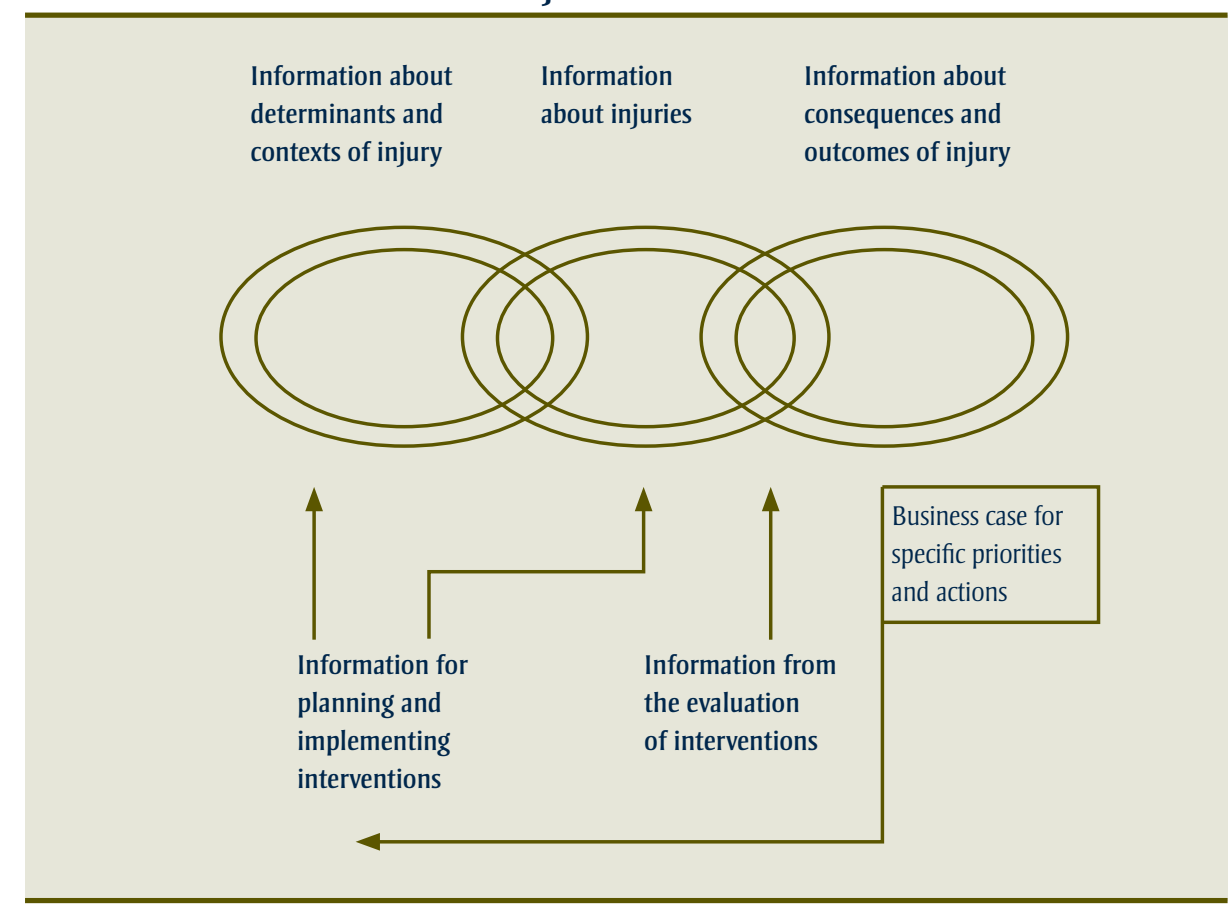

Something that's spatial like this is really effective if you're trying to make a point. It's data that's [sic] being presented in the context of an issue and there's an argument to go along with it and there's, you know, if it's part of a whole package trying to illustrate something, then this kind of spatial representation can be really helpful, because it puts it in context. [\#9]

Finally, informants noted that effective uptake of injury data, however presented, depends on the capacity of organizations like the RHAs. This includes both individual knowledge and skills held by data managers, policy analysts and program developers, and the systems needed to allow these individuals to employ their knowledge to affect practice.

\section{Discussion}

Effective KT requires that research and data be framed to fit the information needs of decision makers. "Researchers tend to describe the past and present with a focus on the 'what.' Decision makers want and need explanation and prediction. They need to know the 'why' and the 'what if'."17 Thus, it is perhaps understandable why
Access to the chain of information will allow key stakeholders to follow forward and backward linkages between determinants, situations, interventions and outcomes. This more complex and holistic view runs against the tendency of researchers and other experts to provide extensive and detailed information about a narrow or circumscribed subject. However, such data may be more costly to collect and more complex to interpret, so there are distinct tradeoffs to be made.

To engage decision makers early in the development of research and determination of data needs would be of key importance, as the literature has argued. ${ }^{4,9,20}$ Published data reports are more likely to be seen as relevant, understood and, perhaps, utilized when there is early and up-front participation of this nature from decision makers. ${ }^{21-22}$

Finally, these informants made the point quite clearly that information in itself is insufficient to have an impact in the policy context. Information needs to fit into a structure that can accommodate and catalyze it. Much literature to date has focused on the interchange between knowledge producers and users at the individual level. ${ }^{23}$ While this is no doubt important, our research also suggests, in line with other recent work on KT, that receptor organizations' systems and structures may determine how well evidence and data can be employed in support of health promotion policy objectives. ${ }^{23-25}$

Our findings are summarized diagrammatically in Figure 2. The injury data that would be valued by these B.C. policy makers make up a chain of information; we have highlighted the interplay between the links in the chain. In our view, this way of looking at KT brings new insight to this field. The nature of decision making is not as linear as this simplified diagram appears, of course. The determinants of injury also likely help shape outcomes or consequences-elderly people who are more prone to injury in certain contexts, such as in long term care facilities, may also have different and less successful outcomes as a result of their age and frailty. Appropriate decisions would also have to account for 
and model such inter-relationships. This suggests a need for multi-level analysis, though again, there are trade-offs between this better representation of the real context in which injuries occur (so important for designing appropriate policy interventions) and the greater demands that such complex analyses place on decision makers' information processing abilities. One of the ongoing objectives of the Burden of Injury in $B C$ project is to explore and develop $\mathrm{KT}$ methods that facilitate effective communication of complex analytic results and uptake of relevant information for policy and priority considerations with respect to injury monitoring, prevention and intervention.

The primary limitation of the current study was the small sample size. It may be that more extensive sampling would have produced other views and further insight into the critical issues around KT and burden-of-injury data. That said, our sampling strategy purposefully included a range of decision makers in different organizations and with different roles and levels of responsibility in injury prevention policy and program development. Convergence of perspectives was observed. However, our informants did not include those identified as data managers, i.e. the persons most likely to handle and interpret detailed data sets on behalf of the RHAs. Further study of the actual practice of injury prevention policy undertaken by RHAs might help indicate how information is actually employed, and whether the detailed data sets asked for here will, in fact, be used or if perhaps decision makers are simply responding to uncertainty or anxiety, or postponing hard choices, by asking for more information.

In looking ahead, further research is required to help elaborate and explain why the data chain that is needed for decision making purposes may be inadequate in the current B.C. policy environment. Is fragmentation the problem or rather the data collection systems? Would progress be advanced through the establishment of a system-wide electronic health record? Working backward from identified needs (as gathered here) to guide systemic reform efforts would be a more grounded approach than current practice, and likely would lead to improved uptake and, ultimately, desired actions at both the individual and system levels.

\section{Conclusion}

In this case study we consulted with decision makers around KT approaches related to one particular set of information: injury data. A number of points raised by the respondents are relevant to doing effective KT. We presume that decision makers would have a similar perspective around other policy areas for which they might be mandated to act, and thus believe these insights are transferable beyond injury data. Nonetheless, testing the information found here, e.g. with other chronic diseases, would be useful. In our view, these findings speak to the relevance of the policy environment and the stages in decision makers' policy cycle that must be understood more fully to ensure adequate uptake and utilization of research knowledge.

\section{References}

1. Landry R, Amara N, Pablos-Mendes A, Shademani R, Gold I. The knowledgevalue chain: a conceptual framework for knowledge translation in health. Bull World Health Organ. 2006;84:597-602.

2. Innvaer S, Vist G, Trommald M, Oxman A. Health policy-makers' perceptions of their use of evidence: a systematic review. J Health Serv Res Policy. 2002;7:239-44.

3. Dobbins M, Cockerill R, Barnsley J, Ciliska, D. Factors of the innovation, organization, environment, and individual that predict the influence five systematic reviews had on public health decisions. Int J Technol Asses. 2001;17:467-78.

4. Bowen S, Martens P, the Need to Know Team. Demystifying knowledge translation: learning from the community. J Health Serv Res Policy. 2005;10:203-11.

5. Lavis J, Ross S, Hurley J, et al. Examining the role of health services research in public policymaking. Milbank Q. 2002;80:125-54.
6. Lavis J, Robertson D, Woodside JM, et al. How can research organizations more effectively transfer research knowledge to decision makers? Milbank Q. 2003;81:221-48.

7. Jacobson N, Butterill D, Goering P. Development of a framework for knowledge translation: understanding user context. J Health Serv Res Policy. 2003;8:94-99.

8. Elliott H, Popay J. How are policy makers using evidence? Models of research utilisation and local NHS policy making. J Epidemiol Commun Health. 2000;54:461-68.

9. Lomas J. Using linkage and exchange to move research into policy at a Canadian foundation. Health Affair. 2000;19:236-40.

10. Roos NP, Shapiro E. From research to policy: what have we learned? Med Care. 1999;27:JS291-JS305.

11. Parry KW. Constant comparison. The Sage encyclopedia of social science research methods. Lewis-Beck MS, Bryman A, Liao TF, editors. Thousand Oaks: Sage, 2004:180-81.

12. British Columbia, Ministry of Health Planning, Office of the Provincial Health Officer. Prevention of falls and injuries among the elderly. Victoria (Canada): Office of the Provincial Health Officer, 2004. Available from: http://www.health.gov.bc.ca/ pho/pdf/falls.pdf.

13. MacNab YC, Kmetic A, Gustafson P, Shaps S. An innovative application of Bayesian disease mapping methods to patient safety research: the Canadian iatrogenic injury study. Stat Med. 2006;25:3960-980.

14. MacNab YC. Mapping disability-adjusted life years: a Bayesian hierarchical model framework for burden of disease and injury assessment. Stat Med. 2007;26:4746-769.

15. Murray CJL, Lopez AD, editors. The global burden of disease. Cambridge (MA): Harvard University Press, 1996.

16. MacNab YC. Spline smoothing in Bayesian disease mapping. Environmetrics. 2007; 18:727-44. 
17. Fraser I. Organizational research with impact: working backwards. Worldviews Evid Based Nurs. 2004;1:S52-S59.

18. Howlett M. Policy development. In The handbook of public administration in Canada. Dunn C, ed. Toronto: Oxford University Press, 2003:173-91.

19. Howlett M, Ramesh M. Studying public policy: policy cycles and policy subsystems. Toronto: Oxford University Press, 1995.

20. Vingilis E, Hartford K, Schrecker T, Mitchell B, Lent B, Bishop J. Integrating knowledge generation with knowledge diffusion and utilization. Can J Public Health. 2003; 94:468-71.

21. Wong K, Gardner S, Bainbridge DB, Feightner K, Offord DR, Chambers LW. Tracking the use and impact of a community social report: where does the information go? Can J Public Health. 2000;91:41-45.

22. Kothari A, Birch S, Charles C. "Interaction" and research utilisation in health policies and programs: does it work? Health Policy. 2005;71:117-25.

23. Hanney S, Gonzalez-Block M, Buxton M, Kogan M. The utilization of health research in policy-making: concepts, examples and methods of assessment. Health Res Policy Syst. 2003 Jan 13;1:2.

24. Reimer B, Sawka E, James D. Improving research transfer in the addictions field: a perspective from Canada. Subst Use Misuse. 2005;40:1707-720.

25. Belkhodja O, Amara N, Landry R, 8 Ouimet M. The extent and organizational determinants of research utilization in Canadian health services organizations. Sci Commun. 2007;28:377-417.

\section{Appendix}

\section{Interview schedule}

1 Please describe your role in relation to injury prevention and control, and/or planning, delivery and policy making pertaining to injury in B.C.

2 Please briefly describe the data and information that you currently access and use in your above-defined role in relation to burden-of-injury in B.C.

3 Are the current data and information that you use adequate?

4 What are the strengths and limitations of the currently available data and information?

5 [Show participant data tables on laptop.] Please refer to the on-screen tables providing detailed information on regional variations in injury-specific mortality, morbidity and burden. [Pause, perhaps 10-15 minutes, to review data.] What are the most relevant data to you in your current role? Why is this the case?

6 Again referring to the data in the tables, what would be the most useful means of presenting this information to you and your colleagues to help ensure that the data are actually used?

7 If these data were made available to you in the manner you have just described, how would you see yourself using this information?

Would you see this information contributing directly to priority setting and resource allocation activity in [the Ministry/your organization]? Please describe how.
9 [Show participant geographical analysis on laptop.] Please refer to the information previously depicted in tabular form now presented in a spatial map on the screen. [Pause, perhaps 10-15 minutes, to review map(s).] Is this a useful depiction of these data, or would you prefer the data in tabular form? Why?

10 Noting that we are developing a knowledge-transfer strategy to assess how this information could be disseminated to policy makers, is there anything else that you could tell us to help us in this process?

Thank you very much for participating in this survey. 